AperTO - Archivio Istituzionale Open Access dell'Università di Torino

\title{
A note on vortices with prescribed charge
}

\section{This is the author's manuscript}

Original Citation:

Availability:

This version is available http://hdl.handle.net/2318/121586

since 2016-01-13T14:35:45Z

Terms of use:

Open Access

Anyone can freely access the full text of works made available as "Open Access". Works made available under a Creative Commons license can be used according to the terms and conditions of said license. Use of all other works requires consent of the right holder (author or publisher) if not exempted from copyright protection by the applicable law. 


\section{(3) \\ UNIVERSITÀ DEGLI STUDI DI TORINO}

This is an author version of the contribution published on:

Questa è la versione dell'autore dell'opera:

A note on vortices with prescribed charge

Advanced Nonlinear studies, vol.12, n.4, 2012

DOI non presente

The definitive version is available at:

La versione definitiva è disponibile alla URL:

http://www.advancednonlinearstudies.com/ANLS_V12N4art.html 


\title{
A note on vortices with prescribed charge*
}

\author{
Marino Badiale - Sergio Rolando \\ Dipartimento di Matematica \\ Università degli Studi di Torino, Via Carlo Alberto 10, 10123 Torino, Italy \\ e-mail: marino.badiale@unito.it, sergio.rolando@unito.it
}

\begin{abstract}
We consider the nonlinear Klein-Gordon equation with nonnegative potential, which makes the equation suitable for physical models, and prove the existence of solitary wave solutions with nonvanishing angular momentum and enough largely prescribed charge (c-vortices). This is done by solving the following minimization problem:

$$
\inf _{(u, \omega) \in H,-\omega\|u\|_{L^{2}\left(\mathbb{R}^{3}\right)}^{2}=c}\left(\frac{1}{2} \int_{\mathbb{R}^{3}}|\nabla u|^{2} d x+\frac{1}{2} \int_{\mathbb{R}^{3}}\left(\frac{k^{2}}{|y|^{2}}+\omega^{2}\right) u^{2} d x+\int_{\mathbb{R}^{3}} V(u) d x\right)
$$

where $x=(y, z) \in \mathbb{R}^{2} \times \mathbb{R}, k \neq 0$ and $H$ is a suitable subspace of $H^{1}\left(\mathbb{R}^{3}\right) \times \mathbb{R}$.
\end{abstract}

\section{Introduction}

In this paper we are concerned with the existence of solitary waves with nonvanishing angular momentum (vortices) and given charge for the nonlinear wave equation

$$
\square \psi+W^{\prime}(\psi)=0,
$$

where $\psi$ is a complex field defined on the spacetime $\mathbb{R}^{4}$, i.e., $\psi(t, x) \in \mathbb{C}$ and $(t, x) \in \mathbb{R} \times \mathbb{R}^{3}$. The operator $\square=\frac{\partial^{2}}{\partial t^{2}}-\triangle$ is the d'Alembert operator and $W^{\prime}(\psi)=V^{\prime}(|\psi|) \frac{\psi}{|\psi|}$ is (under the standard identification between $\mathbb{C}$ and $\mathbb{R}^{2}$ ) the gradient of a function $W: \mathbb{C} \rightarrow \mathbb{R}$ satisfying

$$
W(\psi)=V(|\psi|) \quad \text { for some } V \in C^{2}(\mathbb{R}, \mathbb{R})
$$

Roughly speaking, a solitary wave is a nonsingular solution which travels as a localized packet in such a way that the physical quantities corresponding to the Noether invariances of the equation are finite and conserved in time. Accordingly, solitary waves preserve intrinsic properties of particles such as the energy

$$
\mathcal{E}(\psi)=\int_{\mathbb{R}^{3}}\left[\frac{1}{2}\left|\partial_{t} \psi\right|^{2}+\frac{1}{2}|\nabla \psi|^{2}+W(\psi)\right] d x,
$$

*Work partially supported by the PRIN2009 grant "Critical Point Theory and Perturbative Methods for Nonlinear Differential Equations" 
the angular momentum

$$
\mathbf{M}(\psi)=\operatorname{Re} \int_{\mathbb{R}^{3}} \overline{\partial_{t} \psi}(\mathbf{x} \wedge \nabla \psi) d x
$$

and the charge

$$
\mathcal{C}(\psi)=\operatorname{Im} \int_{\mathbb{R}^{3}} \partial_{t} \psi \bar{\psi} d x,
$$

and can thus be regarded as a model for extended particles, in contrast with point particles. In this respect, they arise in many problems of mathematical physics, such as classical and quantum field theory, nonlinear optics, fluid mechanics, plasma physics and cosmology (see for instance [37, 29, 26]). In addition, the solitary waves of (1.1) exhibit all the most characteristic features of relativistic particles, such as space contraction, time dilation and equivalence between mass and energy (for an introduction to the theory of solitary waves in nonlinear field equations we refer, e.g., to [1, 11, 34]).

Here we are interested in vortices with prescribed charge $c \neq 0$ (in the following, $c$-vortices) for equation (1.1) with nonnegative potentials, that is,

$$
W \geq 0, \quad \mathbf{M}(\psi) \neq \mathbf{0} \quad \text { and } \quad \mathcal{C}(\psi)=c .
$$

Observe that the assumption $W \geq 0$, which implies $\mathcal{E} \geq 0$, is an important requirement for the consistence of physical models related to the equation, since, by the Einstein equation, the existence of field configurations with negative energy would yield negative masses. Furthermore, the positivity of the energy also provides good a priori estimates for the solutions of the corresponding Cauchy problem and these estimates allow to prove that, under very general assumptions on $W$, the problem is well posed (cf. [11]).

The most natural way for finding solitary waves for (1.1) is to look for static waves, i.e., timeindependent solutions of the form $\psi(t, x)=\varphi(x)$, and then to obtain travelling waves by Lorentz transforming. Unfortunately, this forces to assume that $W$ takes negative values, for it is well known, since the renewed paper [22] of Derrik, that $W \geq 0$ implies that any finite-energy static solution of (1.1) is necessarily trivial.

Such a difficulty can be overcome by looking for standing waves, namely, finite-energy solutions having the following form:

$$
\psi(t, x)=\varphi(x) e^{-i \omega t}, \quad \omega \neq 0 .
$$

In the mathematical literature, a lot of work has been done in proving the existence of standing waves with $\varphi(x) \in \mathbb{R}$ (we recall, e.g., [15, 16, 31,32,33]). Also in the physical literature, where the spherically symmetric standing waves are called $Q$-balls according to the name coined by Coleman in [19], there are many papers dealing with this topic, among which we recall the pioneering paper of Rosen [30] and the first rigorous existence paper [18]. In particular, from the results of [15] (see also [11]) it follows that, if $W$ satisfies (1.2) together with

(i) $V(0)=V^{\prime}(0)=0$ and $V \geq 0$

(ii) $V^{\prime \prime}(0)=: \Omega^{2}>0$

(iii) $V\left(s_{0}\right)<\frac{1}{2} \Omega^{2} s_{0}^{2}$ for some $s_{0}>0$,

then equation (1.1) has standing waves (1.6), with $\varphi(x) \in \mathbb{R}$, for every frequency $\omega \in\left(\Omega_{0}, \Omega\right)$, where

$$
\Omega_{0}:=\inf \left\{\omega>0: V(s)<\frac{1}{2} \omega^{2} s^{2} \text { for some } s>0\right\} .
$$


However $\varphi(x) \in \mathbb{R}$ implies $\mathbf{M}(\psi)=\mathbf{0}$ and thus, in order to get vortices, one has to consider complex valued $\varphi$ 's.

Making an ansatz of the form

$$
\psi(t, x)=u(x) e^{i(k \theta(x)-\omega t)}, \quad u(x) \geq 0, \theta(x) \in \frac{\mathbb{R}}{2 \pi \mathbb{Z}}, \omega \neq 0, k \in \mathbb{Z},
$$

equation (1.1) turns out to be equivalent to the system

$$
\left\{\begin{array}{l}
-\triangle u+k^{2}|\nabla \theta|^{2} u-\omega^{2} u+V^{\prime}(u)=0 \\
u \triangle \theta+2 \nabla u \cdot \nabla \theta=0
\end{array}\right.
$$

and, denoting $x=(y, z)=\left(y_{1}, y_{2}, z\right)$, assuming

$$
u(y, z)=u(|y|, z)
$$

and choosing the angular coordinate with respect to the $z$ axis as phase function, i.e., $\theta \in C^{\infty}\left(\mathbb{R}^{3} \backslash \Sigma, \frac{\mathbb{R}}{2 \pi \mathbb{Z}}\right)$ defined by

$$
\theta(x):=\operatorname{Im} \log \left(y_{1}+i y_{2}\right) \quad \text { for every } x \in \mathbb{R}^{3} \backslash \Sigma, \quad \Sigma:=\left\{x \in \mathbb{R}^{3}: y=0\right\},
$$

one gets $\triangle \theta=0, \nabla \theta \cdot \nabla u=0$ and $|\nabla \theta|^{2}=\frac{1}{|y|^{2}}$, so that the above system reduces to

$$
-\triangle u+\frac{k^{2}}{|y|^{2}} u+V^{\prime}(u)=\omega^{2} u .
$$

Direct computations then show that for a field (1.8)-(1.10) the integrals (1.3)-(1.5) become

$$
\begin{gathered}
\mathcal{E}(\psi)=\int_{\mathbb{R}^{3}}\left[\frac{1}{2}|\nabla u|^{2}+\frac{1}{2}\left(\frac{k^{2}}{|y|^{2}}+\omega^{2}\right) u^{2}+V(u)\right] d x, \\
\mathbf{M}(\psi)=\left(0,0,-\omega k \int_{\mathbb{R}^{3}} u^{2} d x\right), \\
\mathcal{C}(\psi)=-\omega \int_{\mathbb{R}^{3}} u^{2} d x
\end{gathered}
$$

(see [2] for a derivation of (1.13)). Hence $\mathbf{M}(\psi)$ does not vanish if $k \neq 0$ and $u \neq 0$. By such arguments, the following results on vortices have been proved in [3] and [4] respectively:

- if $W$ satisfies (1.2) together with (i), (iii) (with $\Omega$ given by (ii*) below) and

(ii*) $\quad V^{\prime}(s)=\Omega^{2} s+O\left(s^{q-1}\right)$ as $s \rightarrow 0^{+}$for some $\Omega>0$ and $q>2$

(ii**) $V^{\prime}(s)=O\left(s^{p-1}\right)$ as $s \rightarrow+\infty$ for some $p<6$,

then equation (1.1) has a nonzero finite-energy classical solution of the form (1.8)-(1.10) for every wave number $k \neq 0$ and every frequency $\omega \in\left(\Omega_{0}, \Omega\right)$, where $\Omega_{0}$ is still given by (1.7) and the limit value $\omega=\Omega$ is also admitted if $q>6$ in (ii*); 
- if $W$ satisfies (1.2) together with (ii*), (iii) and

(i*) $V(0)=V^{\prime}(0)=0$ and $V^{\prime} \geq 0$ on $(0,+\infty)$,

then for every $k \neq 0$ end every $\rho>0$ large enough there exists $\omega \in(0, \Omega]$ such that equation (1.1) has a nonzero finite-energy classical solution of the form (1.8)-(1.10) with $\|u\|_{L^{2}\left(\mathbb{R}^{3}\right)}^{2}=\rho$.

Besides papers [3, 4], the existence of vortices in nonlinear scalar field equations has been also obtained in $[2,5,6,9,13,14,21]$ (see $[7,8,10,12]$ for related results in gauge theories), but the requirement $W \geq 0$ is only permitted in $[5,6]$, where the evolution equations are considered with an additional singular and cylindrical potential, and in [13], where the main theorem contains a weaker result than the above mentioned one from [3] as a particular case. In the physical literature as well, where vortices are called spinning $Q$-balls (even if they are not spherically symmetric), the existence of vortices in classical field theories seems to be an interesting open issue, which has been recently addressed in a number of publications: see for instance [35, 20,17] and the references therein. In particular, the existence of vortices for equation (1.1) has been investigated in [25] and [36], for very particular potentials.

Instead, no results on the problem of $c$-vortices are available in the literature, at least to our knowledge. We observe that the solutions found in [4] have charge $\mathcal{C}(\psi)=-\omega \rho$, which is not known even if $\rho$ is prescribed, because $\omega$ is not known.

Here we prove the following existence (and multiplicity) result on $c$-vortices.

Theorem 1.1. Let $W: \mathbb{C} \rightarrow \mathbb{R}$ satisfy (1.2) and assume

$\left(\mathbf{W}_{\mathbf{1}}\right) V(0)=V^{\prime}(0)=0$ and $V \geq 0$

$\left(\mathbf{W}_{2}\right) V^{\prime}(s)=\Omega^{2} s+O\left(s^{q-1}\right)_{s \rightarrow 0^{+}}$for some $\Omega>0$ and $q>2$

$\left(\mathbf{W}_{3}\right) V\left(s_{0}\right)<\frac{1}{2} \Omega^{2} s_{0}^{2}$ for some $s_{0}>0$

$\left(\mathbf{W}_{4}\right) V^{\prime}(s)=O\left(s^{p-1}\right)_{s \rightarrow+\infty}$ for some $p<10 / 3$.

Fix any $k \in \mathbb{Z}, k \neq 0$. Then for every $|c|$ large enough equation (1.1) has a nonzero classical solution of the form (1.8)-(1.10), satisfying the following properties:

- $\mathcal{E}(\psi)<\infty, \mathbf{M}(\psi)=(0,0, k c)$ and $\mathcal{C}(\psi)=c$;

- $u(y, z)=u(|y|,|z|)$ is nonnegative and nonincreasing in $|z|$.

The assumptions of Theorem 1.1 are satisfied for example by the model potential

$$
W(\psi)=\frac{1}{2} \Omega^{2}|\psi|^{2}-\frac{b}{q}|\psi|^{q}+\frac{1}{p}|\psi|^{p}, \quad \Omega \neq 0,2<q<p<\frac{10}{3},
$$

which is nonnegative provided that $b>0$ is small enough.

Remark 1. Theorem 1.1 also gives travelling $c$-vortices, since, by Lorentz invariance, a solution $\psi_{\mathbf{v}}$ travelling with any vector velocity $\mathbf{v}$ can be obtained from a standing one by boosting. For instance, if $\psi(t, x)=u(x) e^{i(k \theta(x)-\omega t)}$ is a standing $c$-vortex and $\mathbf{v}=(0,0, v),|v|<1$, then

$$
\psi_{\mathbf{v}}(t, x)=u(y, \gamma(z-v t)) e^{i(k \theta(x)-\omega \gamma(t-v z))}, \quad \gamma=\left(1-v^{2}\right)^{-1 / 2},
$$


is also a $c$-vortex, representing a bump which travels in the $z$-direction with speed $v$. Moreover, the same arguments leading to Theorem 1.1 also yield the existence of standing and travelling c-vortices for the nonlinear Schrödinger equation

$$
i \partial_{t} \psi=-\triangle \psi+W^{\prime}(\psi), \quad \psi(t, x) \in \mathbb{C},(t, x) \in \mathbb{R} \times \mathbb{R}^{3} .
$$

Nevertheless, we stated the result for the nonlinear Klein-Gordon equation (1.1) because it is for this equation that, as already mentioned, the assumption $W \geq 0$ has special importance on physical grounds.

We end this introductory section by summarizing the notations of most frequent use throughout the paper.

- We shall always write $x=(y, z) \in \mathbb{R}^{2} \times \mathbb{R}$.

- By $u(y, z)=u(|y|, z)$ we always mean $u(y, z)=u(g y, z)$ for all $g \in O(2)$ (orthogonal group) and almost every $(y, z) \in \mathbb{R}^{2} \times \mathbb{R}$. Similarly for $u(y, z)=u(|y|,|z|)$. The request that $u$ is nonincreasing in $|z|$ then reads as: $\left|z_{1}\right| \leq\left|z_{2}\right| \Rightarrow u\left(y, z_{1}\right) \geq u\left(y, z_{2}\right)$ for almost every $\left(y,\left(z_{1}, z_{2}\right)\right) \in \mathbb{R}^{2} \times \mathbb{R}^{2}$.

- $B_{R}\left(\xi_{0}\right):=\left\{\xi \in \mathbb{R}^{d}:\left|\xi-\xi_{0}\right|<R\right\}$ is the open ball of $\mathbb{R}^{d}$, centered at $\xi_{0}$ and with radius $R$.

- $|A|$ denotes the Lebesgue measure of any measurable set $A \subseteq \mathbb{R}^{d}$.

- By $\rightarrow$ and $\rightarrow$ we respectively mean strong and weak convergence in a Banach space $E$, whose dual space is denoted by $E^{\prime}$. The symbol $\hookrightarrow$ denotes continuous embeddings.

- $C_{\mathrm{c}}^{\infty}(A)$ is the space of the infinitely differentiable real functions with compact support in the open set $A \subseteq \mathbb{R}^{d}$.

- If $1 \leq p \leq \infty$ then $L^{p}(A)$ and $L_{\text {loc }}^{p}(A)$ are the usual Lebesgue spaces (for any measurable set $A \subseteq \mathbb{R}^{d}$ ).

We recall in particular that $u_{n} \rightarrow 0$ in $L_{\text {loc }}^{p}\left(\mathbb{R}^{d}\right)$ if and only if $u_{n} \rightarrow 0$ in $L^{p}\left(B_{R}\right)$ for every $R>0$.

- $H^{1}\left(\mathbb{R}^{3}\right)=\left\{u \in L^{2}\left(\mathbb{R}^{3}\right): \nabla u \in L^{2}\left(\mathbb{R}^{3}\right)\right\}$ is the usual Sobolev space.

\section{A variational principle for $c$-vortices and proof of Theorem 1.1}

In this section, we first show that vortices of charge $c$ can be found as constrained critical points of a suitable functional $E$ on a suitable manifold $\Gamma_{c}$, and then we deduce Theorem 1.1 by solving the minimization problem of $E$ on $\Gamma_{c}$.

Fix any $k \in \mathbb{Z}, k \neq 0$, and define the weighted Sobolev spaces

$$
H:=\left\{u \in H^{1}\left(\mathbb{R}^{3}\right): \int_{\mathbb{R}^{3}} \frac{u^{2}}{|y|^{2}} d x<\infty\right\}, \quad H_{\mathrm{s}}:=\{u \in H: u(y, z)=u(|y|, z)\}
$$

equipped with the hilbertian norm given by

$$
\|u\|^{2}:=\int_{\mathbb{R}^{3}}\left[|\nabla u|^{2}+\left(\frac{k^{2}}{|y|^{2}}+1\right) u^{2}\right] d x \quad \text { for all } u \in H .
$$

Clearly $H_{\mathrm{s}} \hookrightarrow H \hookrightarrow H^{1}\left(\mathbb{R}^{3}\right)$, so that, by well known embeddings of $H^{1}\left(\mathbb{R}^{3}\right)$, one has $H \hookrightarrow L^{p}\left(\mathbb{R}^{3}\right)$ for $2 \leq p \leq 6$ and $H \hookrightarrow L_{\mathrm{loc}}^{p}\left(\mathbb{R}^{3}\right)$ for $1 \leq p \leq 6$. In particular, the latter embedding is compact if $p<6$ and thus it assures that weak convergence in $H$ implies (up to a subsequence) almost everywhere convergence in $\mathbb{R}^{3}$.

Now let $W$ be as in Theorem 1.1. Notice that in the hypotheses of the theorem it is not restrictive to assume $p>2$ and $q<6$. 
For $(u, \omega) \in H \times \mathbb{R}$, we define

$$
\begin{aligned}
& E(u, \omega):=\frac{1}{2} \int_{\mathbb{R}^{3}}\left[|\nabla u|^{2}+\left(\frac{k^{2}}{|y|^{2}}+\omega^{2}\right) u^{2}\right] d x+\int_{\mathbb{R}^{3}} V(u) d x \\
& C(u, \omega):=-\omega \int_{\mathbb{R}^{3}} u^{2} d x, \\
& \Lambda(u, \omega):=\frac{E(u, \omega)}{|C(u, \omega)|} \quad \text { if } C(u, \omega) \neq 0 .
\end{aligned}
$$

Thanks to $\left(\mathbf{W}_{2}\right),\left(\mathbf{W}_{4}\right)$ and the continuous embedding $H \hookrightarrow L^{p}\left(\mathbb{R}^{3}\right) \cap L^{q}\left(\mathbb{R}^{3}\right)$, standard arguments (see for instance [27]) assure that the functional $E$ is of class $C^{1}$ on $H \times \mathbb{R}$ and has Fréchet derivatives $E_{u}^{\prime}(u, \omega) \in H^{\prime}$ and $E_{\omega}^{\prime}(u, \omega) \in \mathbb{R}$ given by

$$
\begin{aligned}
E_{u}^{\prime}(u, \omega) h & =\int_{\mathbb{R}^{3}}\left[\nabla u \cdot \nabla h+\left(\frac{k^{2}}{|y|^{2}}+\omega^{2}\right) u h+V^{\prime}(u) h\right] d x, \\
E_{\omega}^{\prime}(u, \omega) & =\omega \int_{\mathbb{R}^{3}} u^{2} d x .
\end{aligned}
$$

For any given $c \neq 0$, we set

$$
\Gamma_{c}:=\left\{(u, \omega) \in H_{\mathrm{s}} \times \mathbb{R}: C(u, \omega)=c\right\},
$$

which defines a $C^{1}$ manifold in $H_{\mathrm{s}} \times \mathbb{R}$, as $C$ is of class $C^{1}$ on $H \times \mathbb{R}$ and $C_{\omega}^{\prime}(u, \omega)=-\int_{\mathbb{R}^{3}} u^{2} d x \neq 0$ on $\Gamma_{c}$. Notice that $(u, \omega) \in \Gamma_{c}$ implies $u \neq 0$ and $\omega \neq 0$.

Proposition 2.1. Let $c \neq 0$. If $(u, \omega)$ is a critical point of $E$ constrained to $\Gamma_{c}$ and $u$ is nonnegative, then the vortex $\psi(t, x)=u(x) e^{i(k \theta(x)-\omega t)}$ is a finite-energy classical solution of (1.1) with charge $\mathcal{C}(\psi)=c$.

Proof. Let $(u, \omega)$ be as in the assertion. Then there exists a Lagrange multiplier $\lambda \in \mathbb{R}$ such that

$$
\begin{aligned}
E_{u}^{\prime}(u, \omega) h & =\lambda C_{u}^{\prime}(u, \omega) h \quad \text { for all } h \in H_{\mathrm{s}}, \\
E_{\omega}^{\prime}(u, \omega) & =\lambda C_{\omega}^{\prime}(u, \omega) \quad \text { in } \mathbb{R},
\end{aligned}
$$

that is (see $(2.3)-(2.4))$,

$$
\begin{aligned}
-\triangle u+\frac{k^{2}}{|y|^{2}} u+\omega^{2} u+V^{\prime}(u) & =-2 \lambda \omega u \text { in } H_{\mathrm{s}}^{\prime}, \\
\omega \int_{\mathbb{R}^{3}} u^{2} d x & =-\lambda \int_{\mathbb{R}^{3}} u^{2} d x .
\end{aligned}
$$

Since $(u, \omega) \in \Gamma_{c}$ implies $u \neq 0$, the second equation is equivalent to $\lambda=-\omega$ and thus the first equation, which equivalently holds in $H_{\mathrm{s}}^{\prime}$ and $H^{\prime}$ by the Palais' principle of symmetric criticality [28], means that $u$ satisfies

$$
-\triangle u+\frac{k^{2}}{|y|^{2}} u+V^{\prime}(u)=\omega^{2} u \quad \text { in } H^{\prime} .
$$


Then a simple extendibility argument aimed at removing the singularity of $\nabla \theta$ on the plane $y=0$ shows that $\varphi(x)=u(x) e^{i k \theta(x)}$ satisfies

$$
-\triangle \varphi+W^{\prime}(\varphi)=\omega^{2} \varphi
$$

in the distributional sense on $\mathbb{R}^{3}$ (see [3, Lemma 29 and Lemma 30]), so that the standard elliptic regularity theory (see for example [23]) assures that $\varphi$ actually defines a classical solution to (2.6) on $\mathbb{R}^{3}$. A straightforward substitution then shows that the vortex $\psi(t, x)=\varphi(x) e^{-i \omega t}$ classically solves equation (1.1) on $\mathbb{R} \times \mathbb{R}^{3}$. Finally, by definitions of $E$ and $C$, the energy and charge (1.12) and (1.14) of $\psi$ are given by $\mathcal{E}(\psi)=E(u, \omega)<\infty$ and $\mathcal{C}(\psi)=C(u, \omega)=c$.

In order to get critical points of $E$ constrained to $\Gamma_{c}$, we consider problem of minimizing $E(u, \omega)$ on $\Gamma_{c}$. Our result is the following, which is the main result of the paper and will be proved in Section 3.

Theorem 2.2. Let $k \in \mathbb{Z}, k \neq 0$, and let $V \in C^{1}(\mathbb{R}, \mathbb{R})$ be even and satisfying assumptions $\left(\boldsymbol{W}_{1}\right)$-( $\left.\boldsymbol{W}_{4}\right)$ with $\Omega=1$. Then there exists $c_{*}<0$ such that for every $c<c_{*}$ the minimization problem

$$
\inf _{(u, \omega) \in \Gamma_{c}} E(u, \omega)
$$

has a solution $(u, \omega)$, satisfying $u(y, z)=u(|y|,|z|)$ nonnegative and nonincreasing in $|z|$.

Remark 2. In fact, we will prove that the minimization problem (2.7) has a solution ( $u, \omega)$ as in Theorem 2.2 whenever $c<0$ is such that $\inf _{(u, \omega) \in \Gamma_{c}} \Lambda(u, \omega)<1$. For this, we do not need assumption ( $\boldsymbol{W}_{3}$ ) (not even in Lemma 3.5), which only will play a role in Lemma 3.1 in order to assure that the condition $\inf _{(u, \omega) \in \Gamma_{c}} \Lambda(u, \omega)<1$ occurs indeed for $c<0$ large enough.

We can now give the proof of Theorem 1.1, which follows from Theorem 2.2 and Proposition 2.1.

Proof of Theorem 1.1. Fix $k \in \mathbb{Z}, k \neq 0$. In order to apply Theorem 2.2, we first observe that, for any $\Omega>0$, the vortex $\psi(t, x)=u(x) e^{i(k \theta(x)-\omega t)}$ is a finite-energy solution to (1.1) if and only if the vortex

$$
\tilde{\psi}(t, x)=\psi(t / \Omega, x / \Omega)=u(x / \Omega) e^{i(k \theta(x)-\omega t / \Omega)}
$$

is a finite-energy solution to $\square \tilde{\psi}+W^{\prime}(\tilde{\psi}) / \Omega^{2}=0$. Moreover $\mathcal{C}(\tilde{\psi})=\Omega^{2} \mathcal{C}(\psi)$. Hence it is not restrictive to assume $\Omega=1$ in Theorem 1.1, whose hypotheses simultaneously hold for $W$ and $W / \Omega^{2}$. Similarly, there is no loss of generality in proving Theorem 1.1 under the assumption that $V$ is even, since the hypotheses of the theorem simultaneously hold for $V(s)$ and $V(|s|)$. Then, by Theorem 2.2 and Proposition 2.1, there exists $c_{*}<0$ such that for every $c<c_{*}$ the equation (1.1) has a classical solution $\psi(t, x)=$ $u(x) e^{i(k \theta(x)-\omega t)}, u \in H^{1}\left(\mathbb{R}^{3}\right)$, such that $\mathcal{E}(\psi)<\infty, \mathcal{C}(\psi)=c, \mathbf{M}(\psi)=(0,0, k c)$ and $u(y, z)=u(|y|,|z|)$ is nonnegative and nonincreasing in $|z|$. This completes the proof for negative prescribed charges. The case of positive charges then follows by simply changing sign to the frequency of the solution, since $\mathcal{C}\left(u e^{i(k \theta+\omega t)}\right)=-\mathcal{C}\left(u e^{i(k \theta-\omega t)}\right)$

\section{Proof of Theorem 2.2}

In this section we give the proof of Theorem 2.2, which will be achieved through several lemmas. Accordingly, we hereafter assume all the hypotheses of the theorem, supposing $p>2$ and $q<10 / 3$ without restriction. 
The requirement $p, q<10 / 3$ will not be explicitely used in the following and thus it may seem unnecessary. On the contrary, it will be needed in Lemma 3.5, in order to apply the result of $[4$, Theorem $5.1])$.

Lemma 3.1. There exists $c_{*}<0$ such that for every $c<c_{*}$ one has $\inf _{(u, \omega) \in \Gamma_{c}} \Lambda(u, \omega)<1$.

Proof. Here $c_{1}, c_{2}, \ldots$ denote different positive constants, whose precise values will not be relevant. By assumption $\left(\mathbf{W}_{3}\right)$, fix $\alpha>0$ such that $\alpha s_{0}^{2}+V\left(s_{0}\right)-s_{0}^{2} / 2<0$ and set

$$
A:=\left\{(y, z) \in \mathbb{R}^{2} \times \mathbb{R}:|y|>\frac{|k|}{\sqrt{2 \alpha}}\right\} .
$$

Let $R>R_{\alpha}:=1+|k| / \sqrt{2 \alpha}$ and consider two mappings $\varphi_{R}, \psi_{R} \in C^{\infty}((0,+\infty))$ such that

- $\varphi_{R}(t) \equiv s_{0}$ on $(R, 4 R), \varphi_{R}(t) \equiv 0$ on $[0, R-1) \cup(4 R+1,+\infty), 0 \leq \varphi_{R} \leq s_{0}$ on $[0,+\infty)$,

- $\psi_{R}(t) \equiv 1$ on $[0,5 R), \psi_{R}(t) \equiv 0$ on $(5 R+1,+\infty), 0 \leq \psi_{R} \leq 1$ on $[0,+\infty)$,

- $\sup _{R>R_{\alpha}}\left\|\varphi_{R}^{\prime}\right\|_{L^{\infty}((0,+\infty))}<\infty, \sup _{R>R_{\alpha}}\left\|\psi_{R}^{\prime}\right\|_{L^{\infty}((0,+\infty))}<\infty$.

Define $u_{R}(x):=\varphi_{R}(|y|) \psi_{R}(|x|)$ for all $x=(y, z) \in \mathbb{R}^{2} \times \mathbb{R}$. Note that $u_{R} \in C_{\mathrm{c}}^{\infty}(A) \cap H_{\mathrm{s}}$ and $0 \leq u_{R} \leq s_{0}$. Moreover, it is easy to build $\varphi_{R}$ end $\psi_{R}$ in such a way that the mapping $R \in\left(R_{\alpha},+\infty\right) \mapsto u_{R} \in H_{\mathrm{s}}$ is continuous. We will estimate $\Lambda\left(u_{R}, 1\right)$ as $R \rightarrow \infty$. Letting

$$
\begin{aligned}
& A_{1}:=\left\{x \in \mathbb{R}^{3}:|x|<5 R+1, R-1<|y|<4 R+1\right\}, \\
& A_{2}:=\left\{x \in \mathbb{R}^{3}:|x|<5 R, R<|y|<4 R\right\} \subset A_{1}, \\
& A_{4}:=\left\{x \in \mathbb{R}^{3}: 5 R \leq|x| \leq 5 R+1\right\}, \\
& A_{5}:=\left\{x \in \mathbb{R}^{3}:|x|<5 R, R-1 \leq|y| \leq R \text { or } 4 R \leq|y| \leq 4 R+1\right\}, \\
& A_{3}:=A_{1} \backslash A_{2} \subset A_{4} \cup A_{5},
\end{aligned}
$$

one has $u_{R}(x) \equiv 0$ on $\mathbb{R}^{3} \backslash A_{1}, u_{R}(x) \equiv s_{0}$ on $A_{2}$, and $|y|>R-1>R_{\alpha}-1=|k| / \sqrt{2 \alpha}$ on $A_{1}$. Notice that

$$
\begin{aligned}
& \left|A_{4}\right|=c_{1}\left((5 R+1)^{3}-(5 R)^{3}\right)=c_{2} R^{2}+o\left(R^{2}\right)_{R \rightarrow \infty} \\
& \left|A_{5}\right| \leq c_{3}\left(R^{2}-(R-1)^{2}+(4 R+1)^{2}-(4 R)^{2}\right) R=c_{4} R^{2}+o\left(R^{2}\right)_{R \rightarrow \infty} \\
& \left|A_{3}\right| \leq\left|A_{4}\right|+\left|A_{5}\right| \leq c_{5} R^{2}+o\left(R^{2}\right)_{R \rightarrow \infty} \\
& \left|A_{2}\right|=4 \pi \int_{R}^{4 R} \sqrt{25 R^{2}-r^{2}} r d r=\frac{4 \pi}{3}\left(24^{\frac{3}{2}}-9^{\frac{3}{2}}\right) R^{3} \\
& \left|A_{1}\right|=4 \pi \int_{R-1}^{4 R+1} \sqrt{(5 R+1)^{2}-r^{2}} r d r=\frac{4 \pi}{3}\left(\left(24 R^{2}+12 R\right)^{\frac{3}{2}}-\left(9 R^{2}+2 R\right)^{\frac{3}{2}}\right)=\left|A_{2}\right|+o\left(R^{3}\right)_{R \rightarrow \infty}
\end{aligned}
$$

Hence

$$
E\left(u_{R}, 1\right)=\frac{1}{2} \int_{A_{3}}\left|\nabla u_{R}\right|^{2}+\int_{A_{1}}\left(\frac{k^{2}}{2|y|^{2}} u_{R}^{2}+\frac{1}{2} u_{R}^{2}+V\left(u_{R}\right)\right)
$$




$$
\begin{aligned}
& \leq \frac{1}{2} \int_{A_{4} \cup A_{5}}\left|\nabla u_{R}\right|^{2}+\int_{A_{1}}\left(\alpha u_{R}^{2}+V\left(u_{R}\right)-\frac{1}{2} u_{R}^{2}\right)+\int_{A_{1}} u_{R}^{2} d x \\
& \leq c_{6}\left(\left|A_{4}\right|+\left|A_{5}\right|\right)+\int_{A_{2}}\left(\alpha s_{0}^{2}+V\left(s_{0}\right)-\frac{1}{2} s_{0}^{2}\right)+\int_{A_{3}}\left(\alpha u_{R}^{2}+V\left(u_{R}\right)-\frac{1}{2} u_{R}^{2}\right)+s_{0}^{2}\left|A_{1}\right| \\
& \leq\left(\alpha s_{0}^{2}+V\left(s_{0}\right)-\frac{1}{2} s_{0}^{2}\right)\left|A_{2}\right|+c_{7}\left|A_{3}\right|+s_{0}^{2}\left|A_{2}\right|+o\left(R^{3}\right)_{R \rightarrow \infty} \\
& =s_{0}^{2}\left|A_{2}\right|-c_{8} R^{3}+o\left(R^{3}\right)_{R \rightarrow \infty},
\end{aligned}
$$

where we have used the fact that

$$
\left(\alpha s_{0}^{2}+V\left(s_{0}\right)-\frac{1}{2} s_{0}^{2}\right)\left|A_{2}\right|=-c_{8} R^{3},
$$

since $\alpha s_{0}^{2}+V\left(s_{0}\right)-s_{0}^{2} / 2<0$. On the other hand, we have

$$
C\left(u_{R}, 1\right)=-\int_{\mathbb{R}^{3}} u_{R}^{2} d x \leq-\int_{A_{2}} u_{R}^{2} d x=-s_{0}^{2}\left|A_{2}\right| .
$$

Therefore, since $\left|A_{2}\right|=c_{9} R^{3}$, we obtain

$$
\Lambda\left(u_{R}, 1\right)=\frac{E\left(u_{R}, 1\right)}{\left|C\left(u_{R}, 1\right)\right|} \leq \frac{s_{0}^{2} c_{9} R^{3}-c_{8} R^{3}+o\left(R^{3}\right)_{R \rightarrow \infty}}{s_{0}^{2} c_{9} R^{3}}=1-\frac{c_{8}}{s_{0}^{2} c_{9}}+o(1)_{R \rightarrow \infty}
$$

so that one can find $R_{*}>R_{\alpha}$ such that for every $R \geq R_{*}$ it holds

$$
\Lambda\left(u_{R}, 1\right)<1 .
$$

Now observe that the mapping $R \in\left[R_{*},+\infty\right) \mapsto C\left(u_{R}, 1\right) \in \mathbb{R}$ is continuous, since so are the mappings $C: H_{\mathrm{s}} \times \mathbb{R} \rightarrow \mathbb{R}$ and $R \in\left[R_{*},+\infty\right) \mapsto u_{R} \in H_{\mathrm{s}}$. Then, since $\lim _{R \rightarrow \infty} C\left(u_{R}, 1\right)=-\infty$ (see (3.1)), we conclude that for every $c \leq c_{*}:=C\left(u_{R_{*}}, 1\right)<0$ there exists $R \geq R_{*}$ such that $C\left(u_{R}, 1\right)=c$ and $\Lambda\left(u_{R}, 1\right)<1$.

From now till the end of the section, we fix $c<0$ such that $\inf _{(u, \omega) \in \Gamma_{c}} \Lambda(u, \omega)<1$ (which exists by Lemma 3.1) and set

$$
\nu_{c}:=\inf _{(u, \omega) \in \Gamma_{c}} E(u, \omega) .
$$

Notice that $(u, \omega) \in \Gamma_{c}$ implies $u \neq 0$ and $\omega>0$.

Lemma 3.2. The minimizing sequences of (3.2) are bounded in $H \times \mathbb{R}$.

Proof. Let $\left\{\left(u_{n}, \omega_{n}\right)\right\}$ be a minimizing sequence of (3.2). Then $\omega_{n}>0$ and $\omega_{n} \int_{\mathbb{R}^{3}} u_{n}^{2} d x=|c|$, so that

$$
E\left(u_{n}, \omega_{n}\right)=\frac{1}{2} \int_{\mathbb{R}^{3}}\left(\left|\nabla u_{n}\right|^{2}+\frac{k^{2}}{|y|^{2}} u_{n}^{2}\right) d x+\frac{1}{2}|c| \omega_{n}+\int_{\mathbb{R}^{3}} V\left(u_{n}\right) d x .
$$

Since $\left\{E\left(u_{n}, \omega_{n}\right)\right\}$ is bounded and $V \geq 0$ by assumption $\left(\mathbf{W}_{1}\right)$, we readily get that $\left\{\omega_{n}\right\}$ and $\left\{\left\|u_{n}\right\|^{2}-\right.$ $\left.\left\|u_{n}\right\|_{L^{2}\left(\mathbb{R}^{3}\right)}^{2}\right\}$ are bounded, so that we need only to prove that $\left\{\left\|u_{n}\right\|_{L^{2}\left(\mathbb{R}^{3}\right)}\right\}$ is bounded. To this aim, we 
observe that $V(0)=0$ and assumption $\left(\mathbf{W}_{2}\right)$, together with the evenness of $V$, imply that there exists $\delta>0$ such that $V(s) \geq \frac{1}{4} s^{2}$ for $|s| \leq \delta$, so that for all $n$ we get

$$
\begin{aligned}
\int_{\mathbb{R}^{3}} u_{n}^{2} d x & =\int_{\left\{\left|u_{n}\right| \leq \delta\right\}} u_{n}^{2} d x+\int_{\left\{\left|u_{n}\right|>\delta\right\}} u_{n}^{2} d x \leq 4 \int_{\mathbb{R}^{3}} V\left(u_{n}\right) d x+\int_{\left\{\left|u_{n}\right|>\delta\right\}} u_{n}^{2}\left(\frac{\left|u_{n}\right|}{\delta}\right)^{4} d x \\
& \leq 4 E\left(u_{n}, \omega_{n}\right)+\frac{1}{\delta^{4}} \int_{\mathbb{R}^{3}} u_{n}^{6} d x .
\end{aligned}
$$

By Sobolev inequality, this implies that $\left\{\left\|u_{n}\right\|_{L^{2}\left(\mathbb{R}^{3}\right)}\right\}$ is bounded and the proof is complete.

For computational convenience, we henceforth denote

$$
J(u):=\frac{1}{2} \int_{\mathbb{R}^{3}}\left(|\nabla u|^{2}+\frac{k^{2}}{|y|^{2}} u^{2}-u^{2}\right) d x+\int_{\mathbb{R}^{3}} V(u) d x
$$

and for any $\rho>0$ we set

$$
m_{\rho}:=\inf _{u \in \mathcal{M}_{\rho}} J(u), \quad \mathcal{M}_{\rho}:=\left\{u \in H_{\mathrm{s}}: \int_{\mathbb{R}^{3}} u^{2} d x=\rho\right\} .
$$

Then for every $(u, \omega) \in H \times \mathbb{R}$ we have

$$
E(u, \omega)=J(u)+\frac{\omega^{2}}{2} \int_{\mathbb{R}^{3}} u^{2} d x+\frac{1}{2} \int_{\mathbb{R}^{3}} u^{2} d x=J(u)-\frac{\omega}{2} C(u, \omega)+\frac{1}{2} \int_{\mathbb{R}^{3}} u^{2} d x .
$$

By Lemma 3.2, we now fix a minimizing sequence of (3.2) such that

$$
\begin{array}{ll}
\omega_{n} \rightarrow \omega_{0}, & \left\|u_{n}\right\|_{L^{2}\left(\mathbb{R}^{3}\right)}^{2} \rightarrow \rho, \\
u_{n} \rightarrow u_{0} \text { in } H_{\mathrm{s}}, & 0 \leq u_{n} \rightarrow u_{0} \text { in } L_{\mathrm{loc}}^{r}\left(\mathbb{R}^{3}\right) \text { for } 1 \leq r<6 .
\end{array}
$$

Notice that $\omega_{0} \neq 0$ and $\rho \neq 0$, since $\left(u_{n}, \omega_{n}\right) \in \Gamma_{c}$ implies

$$
\omega_{0} \rho=\lim _{n \rightarrow \infty} \omega_{n} \int_{\mathbb{R}^{3}} u_{n}^{2} d x=|c| \neq 0
$$

Lemma 3.3. One has

$$
\nu_{c} \leq m_{\rho}+\frac{1}{2} \rho+\frac{c^{2}}{2 \rho}
$$

Proof. For every $v \in \mathcal{M}_{\rho}$, we have $C(v,|c| / \rho)=-|c|=c$ and thus, by (3.4), we get

$$
\nu_{c} \leq E\left(v, \frac{|c|}{\rho}\right)=J(v)+\frac{1}{2} \frac{c^{2}}{\rho}+\frac{1}{2} \rho .
$$

Hence the claim follows, by taking the infimum as $v \in \mathcal{M}_{\rho}$.

Lemma 3.4. One has

$$
\nu_{c} \geq m_{\rho}+\frac{1}{2} \rho+\frac{c^{2}}{2 \rho}
$$


Proof. By (3.4) and (3.5), the minimizing sequence $\left(u_{n}, \omega_{n}\right)$ satisfies

$$
E\left(u_{n}, \omega_{n}\right)=J\left(u_{n}\right)+\frac{1}{2}|c| \omega_{n}+\frac{1}{2} \int_{\mathbb{R}^{3}} u_{n}^{2} d x=J\left(u_{n}\right)+\frac{1}{2}|c| \omega_{0}+\frac{1}{2} \rho+o(1)_{n \rightarrow \infty},
$$

so that, by (3.6), we get

$$
J\left(u_{n}\right) \rightarrow \nu_{c}-\frac{c^{2}}{2 \rho}-\frac{1}{2} \rho
$$

We now show that $v_{n}:=\sqrt{\omega_{n} / \omega_{0}} u_{n}$ satisfies $J\left(v_{n}\right)-J\left(u_{n}\right) \rightarrow 0$ and belongs to $\mathcal{M}_{\rho}$, which clearly concludes the proof. By the definition of $J$ and using (3.5) and Lemma 3.2, we get

$$
\begin{aligned}
\left|J\left(v_{n}\right)-J\left(u_{n}\right)\right| & \leq \frac{1}{2}\left|\frac{\omega_{n}}{\omega_{0}}-1\right| \int_{\mathbb{R}^{3}}\left(\left|\nabla u_{n}\right|^{2}+\frac{k^{2}}{|y|^{2}} u_{n}^{2}+u_{n}^{2}\right) d x+\int_{\mathbb{R}^{3}}\left|V\left(v_{n}\right)-V\left(u_{n}\right)\right| d x \\
& =o(1)_{n \rightarrow \infty}+\int_{\mathbb{R}^{3}}\left|V^{\prime}\left(\xi_{n}\right)\right|\left|v_{n}-u_{n}\right| d x=o(1)_{n \rightarrow \infty}\left(1+\int_{\mathbb{R}^{3}}\left|V^{\prime}\left(\xi_{n}\right)\right|\left|u_{n}\right| d x\right)
\end{aligned}
$$

where

$$
\xi_{n}=\vartheta_{n} v_{n}+\left(1-\vartheta_{n}\right) u_{n}=\left(1+\vartheta_{n}\left(\sqrt{\frac{\omega_{n}}{\omega_{0}}}-1\right)\right) u_{n}
$$

for some $0 \leq \vartheta_{n} \leq 1$ (recall that $V$ is of class $C^{1}$ ). Observe that $\left|\xi_{n}\right|=\left(1+o(1)_{n \rightarrow \infty}\right)\left|u_{n}\right|$. Now we use assumptions $\left(\mathbf{W}_{2}\right)$ and $\left(\mathbf{W}_{4}\right)$, which, together with the continuity and the evenness of $V$, imply the existence of some constant $c_{0}>0$ such that

$$
\left|V^{\prime}(s)\right| \leq c_{0}\left(|s|+|s|^{p-1}+|s|^{q-1}\right) \quad \text { for all } s \in \mathbb{R}
$$

Therefore one has

$$
\left|V^{\prime}\left(\xi_{n}\right)\right|\left|u_{n}\right| \leq c_{0}\left(\left|\xi_{n}\right|+\left|\xi_{n}\right|^{p-1}+\left|\xi_{n}\right|^{q-1}\right)\left|u_{n}\right|=\left(c_{0}+o(1)_{n \rightarrow \infty}\right)\left(u_{n}^{2}+\left|u_{n}\right|^{p}+\left|u_{n}\right|^{q}\right),
$$

which implies that

$$
\int_{\mathbb{R}^{3}}\left|V^{\prime}\left(\xi_{n}\right)\right|\left|u_{n}\right| d x \text { is bounded, }
$$

since $\left\{u_{n}\right\}$ is bounded in $H$ (Lemma 3.2) and $H \hookrightarrow L^{p}\left(\mathbb{R}^{3}\right) \cap L^{q}\left(\mathbb{R}^{3}\right)$ because $p, q \in(2,6)$. This proves that $\left|J\left(v_{n}\right)-J\left(u_{n}\right)\right| \rightarrow 0$. Finally, by (3.6) we have

$$
\int_{\mathbb{R}^{3}} v_{n}^{2} d x=\frac{\omega_{n}}{\omega_{0}} \int_{\mathbb{R}^{3}} u_{n}^{2} d x=\frac{|c|}{\omega_{0}}=\rho
$$

which means $v_{n} \in \mathcal{M}_{\rho}$.

In order to conclude the proof of Theorem 2.2, we need the following result from [4] (see also [24]).

Lemma 3.5. If $\rho>0$ is such that $m_{\rho}<0$, then the minimization problem (3.3) has a solution $u(y, z)=$ $u(|y|,|z|) \geq 0$ which is nonincreasing in $|z|$. 
Proof. It is Theorem 5.1 of [4], whose assumptions hold true for $F(s)=\frac{1}{2} s^{2}-V(s), f(s)=F^{\prime}(s)$, thanks to $\left(\mathbf{W}_{2}\right)-\left(\mathbf{W}_{4}\right)$ together with $V(0)=0$ and the continuity and evenness of $V$.

Proof of Theorem 2.2. First observe that $\rho \neq 0$ and $\rho^{2}-2|c| \rho+c^{2}=(\rho-|c|)^{2} \geq 0$ imply

$$
\frac{1}{2} \rho+\frac{c^{2}}{2 \rho} \geq|c| .
$$

Then from Lemma 3.1 we deduce $\nu_{c}<|c|\left(\right.$ take $(\bar{u}, \bar{\omega}) \in \Gamma_{c}$ such that $\left.E(\bar{u}, \bar{\omega})<|C(\bar{u}, \bar{\omega})|=|c|\right)$, so that (3.7) and Lemmas 3.3 and 3.4 yield

$$
m_{\rho}+|c| \leq m_{\rho}+\frac{1}{2} \rho+\frac{c^{2}}{2 \rho}=\nu_{c}<|c| .
$$

Hence we get $m_{\rho}<0$ and thus Lemma 3.5 assures that there exists a nonnegative $u \in \mathcal{M}_{\rho}$ such that $u$ is radial and nonincreasing in $|z|$ and $J(u)=m_{\rho}$. Therefore, by definition of $C$, we get

$$
C\left(u, \frac{|c|}{\rho}\right)=-\frac{|c|}{\rho}\|u\|_{L^{2}\left(\mathbb{R}^{3}\right)}^{2}=-|c|=c,
$$

so that, by (3.4) and Lemmas 3.3 and 3.4 again, we conclude

$$
E\left(u, \frac{|c|}{\rho}\right)=J(u)-\frac{|c|}{2 \rho} C\left(u, \frac{|c|}{\rho}\right)+\frac{1}{2} \int_{\mathbb{R}^{3}} u^{2} d x=m_{\rho}+\frac{c^{2}}{2 \rho}+\frac{1}{2} \rho=\nu_{c} .
$$

This proves that $(u,|c| / \rho)$ attains the infimum (2.7), since (3.8) means $(u,|c| / \rho) \in \Gamma_{c}$.

\section{References}

[1] Badiale M., Benci V., Rolando S., Solitary waves: physical aspects and mathematical results, Rend. Sem. Math. Univ. Pol. Torino 62 (2004), 107-154.

[2] Badiale M., Benci V., Rolando S., A nonlinear elliptic equation with singular potential and applications to nonlinear field equations, J. Eur. Math. Soc. 9 (2007), 355-381.

[3] Badiale M., Benci V., Rolando S., Three dimensional vortices in the nonlinear wave equation, Bollettino U.M.I., Serie 9, II (2009), 105-134.

[4] Badiale M., Rolando S., Vortices with prescribed $L^{2}$ norm in the nonlinear wave equation, Adv. Nonlinear Stud. 8 (2008), 817-842.

[5] Badiale M., Rolando S., Nonlinear elliptic equations with subhomogeneous potentials, Nonlinear Anal. 72 (2010), 602-617.

[6] Bellazzini J., Bonanno C., Nonlinear Schrodinger equations with strongly singular potentials, Proc. Roy. Soc. Edinburgh A 140 (2010), 707-721.

[7] Bellazzini J., Bonanno C., Siciliano G., Magneto-static vortices in two dimensional Abelian gauge theories, Mediterr. J. Math. 6 (2009), 347-366. 
[8] Benci V., Bonanno C., Solitary waves and vortices in non-Abelian gauge theories with matter, preprint 2011.

[9] Benci V., D'Aprile T., The semiclassical limit of the nonlinear Schrödinger equation in a radial potential, J. Differential Equations 184 (2002), 109-138.

[10] Benci V., Fortunato D., Existence of 3D-vortices in abelian gauge theories, Mediterr. J. Math. 3 (2006), 409-418.

[11] Benci V., Fortunato D., Solitary waves in the nonlinear wave equation and in gauge theories, J. Fixed Point Theory Appl. 1 (2007), 61-86.

[12] Benci V., Fortunato D., Three dimensional vortices in abelian gauge theories, Nonlinear Anal. 70 (2009), 4402-4421.

[13] Benci V., Fortunato D., Spinning Q-balls for the Klein-Gordon-Maxwell equations, Comm. Math. Phys. 295 (2010), 639-668.

[14] Benci V., Visciglia N., Solitary waves with non vanishing angular momentum, Adv. Nonlinear Stud. 3 (2003), 151-160.

[15] Berestycki H., Lions P.L., Nonlinear scalar field equations, I - Existence of a ground state, Arch. Rational Mech. Anal. 82 (1983), 313-345.

[16] Berger M.S., On the existence and structure of stationary states for a nonlinear Klein-Gordon equation, J. Funct. Analysis 9 (1972), 249-261.

[17] Brihaye Y., Hartmann B., Zakrzewski W.J., Spinning solitons of a modified non-linear Schroedinger equation, Phys. Rev. D 69, 087701.

[18] Coleman S., Glaser V., Martin A., Action minima among solutions to a class of euclidean scalar field equation, Comm. Math. Phys 58 (1978), 211-221.

[19] Coleman S., Q-Balls, Nucl. Phys. B 262 (1985), 263-283.

[20] Crasovan L.C., Malomed B.A., Mihalache D., Spinning solitons in cubic-quintic nonlinear media, Pramana 57 (2001), 1041- 1059.

[21] D'Aprile T., On a class of solutions with non-vanishing angular momentum for nonlinear Schrödinger equations, Diff. Integral Eq. 16 (2003), 349-384.

[22] DerRick G.H., Comments on nonlinear wave equations as models for elementary particles, J. Math. Phys. 5 (1964), 1252-1254.

[23] Giaquinta M., Introduction to regularity theory for nonlinear elliptic systems, Birkhäuser Verlag, 1993.

[24] Jeanjean L., Squassina M., An approach to minimization under a constraint: the added mass technique, Calc. Var. Partial Differ. Equ. 41 (2011), 511-534.

[25] Kiм C., Kıм S., Kiм Y., Global nontopological vortices, Phys. Rev. D 47 (1985), 5434-5443. 
[26] Kusenko A., Shaposhnikov M., Supersymmetric Q-balls as dark matter, Phys. Lett. B 418 (1998), 46-54.

[27] Kuzin I., Pohožaev S., Entire solutions of semilinear elliptic equations, PNLDE, vol. 33, Birkhäuser, 1997.

[28] Palais R.S., The principle of symmetric criticality, Commun. Math. Phys. 69 (1979), 19-30.

[29] Rajaraman R., Solitons and instantons, North-Holland Physics Publishing, 1987.

[30] Rosen G., Particlelike solutions to nonlinear complex scalar field theories with positive-definite energy densities, J. Math. Phys. 9 (1968), 996-998.

[31] Shatah J., Stable standing waves of nonlinear Klein-Gordon equations, Comm. Math. Phys. 91 (1983), 313-327.

[32] Sнатан J., Unstable ground state of nonlinear Klein-Gordon equations, Trans. Amer. Math. Soc. 290 (1985), 701-710.

[33] Strauss W.A., Existence of solitary waves in higher dimensions, Comm. Math. Phys. 55 (1977), $149-172$.

[34] Strauss W.A., Nonlinear invariant wave equations, Lecture Notes in Physics, vol. 23, Springer, 1978.

[35] Volkov M.S., Existence of spinning solitons in field theory, eprint arXiv:hep-th/0401030 (2004).

[36] Volkov M.S., Wöhnert E., Spinning Q-balls, Phys. Rev. D 66 (2002) 085003.

[37] Wiтнам G.B., Linear and nonlinear waves, John Wiley \& Sons, 1974.

Marino Badiale, Sergio Rolando

Dipartimento di Matematica

Università degli Studi di Torino

Via Carlo Alberto 10

10123 Torino, Italia

marino.badiale@unito.it, sergio.rolando@unito.it

Work partially supported by the PRIN2009 grant "Critical Point Theory and Perturbative Methods for Nonlinear Differential Equations" 